\title{
Carbon-ion radiotherapy for non-small cell lung cancer with interstitial lung disease: a retrospective analysis
}

\author{
Mio Nakajima ${ }^{1 *}$ D, Naoyoshi Yamamoto ${ }^{1}$, Kazuhiko Hayashi ${ }^{1}$, Masataka Karube ${ }^{1,2}$, Daniel K Ebner ${ }^{1,3}$, \\ Wataru Takahashi ${ }^{1,2}$, Makoto Anzai ${ }^{4}$, Kenji Tsushima ${ }^{5}$, Yuji Tada ${ }^{5}$, Koichiro Tatsumi ${ }^{5}$, Tadaaiki Miyamoto ${ }^{1}$, \\ Hiroshi Tsuji ${ }^{1}$, Takehiko Fujisawa ${ }^{6}$ and Tadashi Kamada'
}

\begin{abstract}
Background: Lung cancer is frequently complicated by interstitial lung disease (ILD). Treatment protocols for lung cancer patients with ILD have not been established; surgery, chemotherapy, and radiotherapy can all cause acute exacerbation of ILD. This study evaluated the toxicity and efficacy of carbon ion radiotherapy (CIRT) in patients with non-small cell lung cancer (NSCLC) and ILD.

Methods: Between June 2004 and November 2014, 29 patients diagnosed with NSCLC and ILD were treated with CIRT. No patient was eligible for curative surgery or conventional radiotherapy secondary to ILD. Owing to prior symptomology, radiation pneumonitis (RP) and symptom progression pre- and post-treatment were evaluated. The relationships between RP and clinical factors were investigated.

Results: Twenty-eight men and one woman, aged 62 to 90 years old, were followed for 2.7-77.1 months (median: 22.8 months). Single-grade symptomatic progression (grade 2-3) was observed in 4 patients, while 1 patient experiencedtwo-grade progression. Two patients experienced radiation-induced acute exacerbation. Local control at 3 years was $63.3 \%$ ( $72.2 \%$ for stage I disease); survival at 3 years was $46.3 \%$ (57.2\% for stage I disease). Eighteen patients had died by the time of this writing, 10 of lung cancer progression. Radiation pneumonitis post-treatment progression correlated with dosimetric factors of the lungs $(\mathrm{V} 5, \mathrm{~V} 10)$ and a low pre-treatment serum surfactant protein-D.
\end{abstract}

Conclusions: We found that CIRT may be useful as a low-risk, curative option for NSCLC patients with ILD, a population that is typically ineligible for conventional therapy. The DVH analysis showed that minimizing the low-dose region is important for reducing the risk of severe RP.

Trial registration: NIRS-9404. Registered 1 March 1994.

Keywords: Carbon ion radiotherapy, Lung cancer, Interstitial lung disease, Radiotherapy, Radiation pneumonitis

\section{Background}

Lung cancer (LC) is often associated with interstitial lung disease (ILD). The reported prevalence of LC in ILDs is $13-50 \%$, particularly in the setting of interstitial pulmonary fibrosis (IPF) [1-4].

Treatment protocols for ILD-associated LC have not been well-established, as surgery, chemotherapy, and

\footnotetext{
* Correspondence: nakajima.mio@qst.go.jp

${ }^{1}$ National Institute of Radiological Sciences Hospital, National Institutes for Quantum and Radiological Sciences and Technology, 4-9-1, Anagawa, Inage-ward, Chiba 263-8555, Japan

Full list of author information is available at the end of the article
}

radiotherapy can all cause acute exacerbation (AE) of ILD. The postoperative $\mathrm{AE}$ frequency has been reported to be $9-25 \%$; with a mortality rate of $11-86 \%$, it accounts for the majority of treatment-related deaths [5-9].

Stereotactic body radiation therapy (SBRT) is a noninvasive cancer treatment, and has proven to be an effective and well-tolerated treatment in numerous studies for early stage LC in medically inoperable patients [10-12]. However, severe ILD is considered a relative contraindication in the clinical guidelines for SBRT published by the Japanese Society for Therapeutic Radiation and Oncology, as a Japanese national survey of SBRT found that most patients 
with pulmonary grade 5 radiation pneumonitis (RP) had ILD [13]. High post-treatment mortality, even with mild ILD, has caused great difficulty in designing treatments for ILD-LC patients, and treatment results remain unsatisfactory [14].

In 1994, the National Institute of Radiological Sciences (NIRS) began exploring carbon-ion radiotherapy (CIRT) as a treatment for non-small cell lung cancer (NSCLC). Carbon-ion beams offer unique treatment advantages compared to other radiation modalities, including the delivery of a highly precise dose concentration to the target, with high linear energy transfer and relative biological effectiveness throughout the spread-out Bragg peak [15]. As reported previously, hypofractionated CIRT has produced excellent local control rates that are comparable to those of surgery or SBRT, with markedly low toxicities [16-19].

In this study, we evaluated the toxicity and efficacy of CIRT for treating NSCLC in patients also presenting with ILD.

\section{Methods}

\section{Study design and patient selection}

We performed a retrospective evaluation of all patients with ILD-LC who were treated with CIRT at our hospital. Owing to the presense of ILD, all enrolled patients were ineligible for curative surgery and conventional radiotherapy.We diagnosed ILD was according to medical history, physical examination, respiratory function test, and evaluation of abnormalities compatible with bilateral lung fibrosis on chest computed tomography (CT) or high-resolution CT, such as peripheral reticular opacities. Biochemical tests for levels of lactate dehydrogenase, surfactant protein-D (SP-D), and serum Krebs von den Lungen-6 (KL-6) were also performed.

The treatment method and procedure were approved by the ethics committees of our institute, and written informed consent was obtained from all patients included in the study.

\section{Lung cancer staging and interstitial lung disease severity}

Initial workup included clinical and laboratory examinations, contrast-enhanced CT of the chest, contrastenhanced magnetic resonance imaging (MRI) of the brain, and $[11 \mathrm{C}]$-acetate or $[18 \mathrm{~F}]$-fluorodeoxyglucose $\left({ }^{18} \mathrm{~F}-\mathrm{FDG}\right)$ positron emission tomography (PET)/CT scanning for detecting involved lymph nodes and distant metastases. The severity of ILD was evaluated according to the criteria of the Japanese Respiratory Society classifications [20].

\section{Carbon-ion radiotherapy procedure}

Carbon ion beams with 290, 350, and $400 \mathrm{MeV}$ of nucleon energy were generated in the Heavy Ion Medical Accelerator in Chiba (HIMAC) synchrotron and delivered to the treatment room. The details of CIRT planning and delivery at our institution have been described previously [16, $18,19,21]$.

Between June 2004 and November 2014, CIRT was performed on 637 patients with primary NSCLC lesions at our institution; among them, 29 patients had ILD. Two patients were diagnosed with hilar lymph node metastasis. Three patients had suspected mediastinal lymph node metastasis on PET/CT, and 1 had suspected accessory nerve lymph node metastasis. We treated only the primary lesions of these patients, as Konishi et al. and Roberts et al. reported that inflammatory pulmonary lesions may show increased uptake of 18F-FDG [22, 23]. Additionally, 1 patient was diagnosed with an intrapulmonary metastatic lesion near their primary tumor. Both primary and metastatic lesions were treated in the same field.

For stage I LC patients and 4 patients with suspected lymph node metastases, the primary tumors were irradiated with carbon-ion beams at a fixed dose of 52.8 Gy (relative biological effectiveness [RBE]) (13.2 Gy[RBE]/fraction) for $\mathrm{T} 1 \quad(\leq 3 \mathrm{~cm})$ tumors and 60.0 Gy (RBE) (15 Gy[RBE]/fraction) for T2 $(>3 \mathrm{~cm})$ tumors, delivered in 4 fractions over the course of 1 week. A single T4 patient was included in the T2 tumor group; this patient's primary tumor and nearby intrapulmonary metastatic lesion were irradiated. After February 2012, primary tumors in stage I LC patients were irradiated in a single fraction at doses prescribed by the NIRS Lung Cancer Single Fraction Clinical Trial [19]. For single fraction delivery, tumors were normally irradiated from 4 ports consecutively. The HIMAC delivery system allowed for patients to be rotated a maximum of \pm 20 degrees, allowing for four different treatment angles between the horizontal and vertical particle beams.

In accordance with routine practice at our institute for stage I NSCLC, all CIRT plans utilized 4 coplanar and oblique beam fields at mutual angles of 40 or 50 degrees.

For two patients with stage II NSCLC who had hilar lymph node metastases, the primary tumor and involved lymph nodes were contoured as the gross tumor volume. The clinical target volume included the primary tumor with 10-mm margins in all directions. Prophylactic regional nodal irradiation that included targeting the ipsilateral hilar and mediastinal lymph nodes was chosen for one of the patients with a hilar lymph node metastasis. For the other patient, only the primary tumor and involved lymph node were irradiated. The prescribed dose (72 Gy [RBE]) was administered to the primary tumor, and a reduced dose was delivered to the CTV up to a total dose of $50 \mathrm{~Gy}$ (RBE) at the discretion of the treating physician. A fixed dose of 72 Gy (RBE) was delivered over a course of 16 daily fractions administered on four consecutive days per week and over four consecutive weeks.

The planning target volume (PTV) was enclosed by the $95 \%$ isodose line for conformal treatment with the 
prescribed dose. Dose constraints were strictly adhered to, regardless of potential target coverage compromise, as follows: spinal cord, 30 Gy (RBE); esophagus, 50 Gy (RBE); and mainstem bronchus, 60 Gy (RBE). Figure 1 shows a sample of the dose distribution for a patient wih stage I disease.

\section{Follow-up}

After the end of radiotherapy, regular follow-up examinations were performed every 3 months for the first 2 years and every 6 months thereafter. Each follow-up session included a physical evaluation, chest CT, brain MRI, respiratory function test, and laboratory tests at a minimum. Additional imaging investigations such as bone scanning and PET/CT were performed upon clinical suspicion of recurrence.

\section{Radiation pneumonitis grading and the definition of acute exacerbation}

The severity of RP was graded according to National Cancer Institute Common Terminology Criteria for Adverse Events (CTCAE), version 3.0. As many patients had respiratory symptoms prior to CIRT, symptoms were graded before and after CIRT, and symptomatic grade change was evaluated. The occurrence of $\mathrm{AE}$ was defined according to the revised Japanese criteria for $\mathrm{AE}$ of IPF [20], which states that all of the following three conditions must be satisfied during the course of IPF within a single month: 1) worsening or development of dyspnea 2) new ground-glass opacities appear on high-resolution $\mathrm{CT}$ in addition to previous honeycomb lesions, new ground-glass abnornalities, and/or consolidation superimposed on a background reticular or honeycomb pattern on high-resolution CT 3) a greater than $10 \mathrm{mmHg}$ reduction of partial pressure of oxygen in the arterial blood under similar conditions.

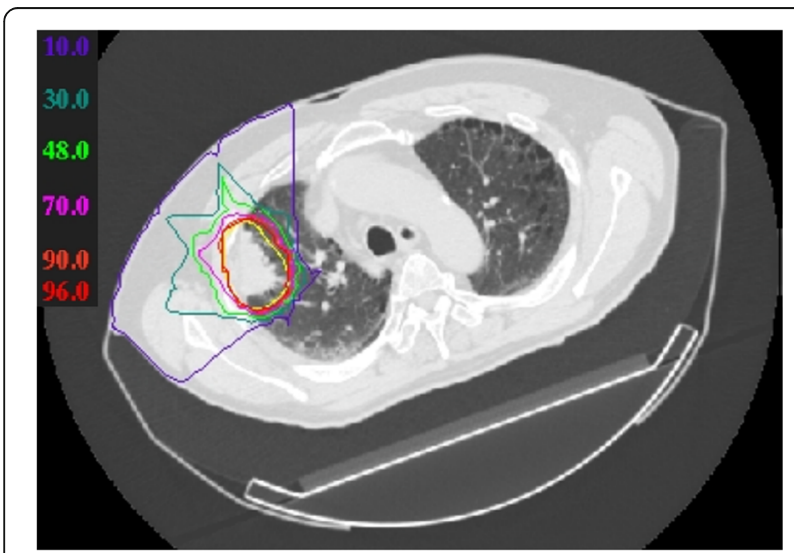

Fig. 1 Carbon-ion dose distribution in a patient with stage I nonsmall cell lung cancer. The yellow and red lines indicate clinical target volume and $95 \%$ isodose of the prescribed dose, respectively
The ruling out of alternative causes, such as infection, pneumothorax, cancer, pulmonary embolism or congestive heart failure is required. Due to the difficulty of distinguishing $\mathrm{AE}$ from $\mathrm{RP}, \mathrm{AE}$ was confirmed when infiltrates were observed outside the radiation field.

\section{Statistical analysis}

All data are presented as median values with ranges. We used JMP statistical software (version 11.0) for all statistical analyses. To investigate the relationships between patients with or without IP grade progression, clinical characteristics and treatment-related factors were compared using the Mann-Whitney U test or Fisher's exact test.

Overall survival was defined as the time elapsed from the start of CIRT to death or to last follow-up date (i.e., last hospital visit, phone call, or mailed communication). Patients lost to follow-up or who were alive at the end of the study were censored. The local control rate was calculated from the date of initial CIRT to the first local recurrence was first detected, or that of the patient's last follow-up visit. The overall survival and local control rates were analyzed using Kaplan-Meier statistics. Cox proportional hazards analysis was conducted to identify prognostic factors. A $p$-value less than 0.05 was considered statistically significant. Any AEs occurring after radiotherapy were evaluated qualitatively on an individual basis due to their low numbers.

\section{Results}

Patient characteristics and the severity of interstitial lung disease

In total, 637 consecutive patients with NSCLC who underwent CIRT between June 2004 and November 2014 were investigated retrospectively. Of these, 29 patients with ILD were identified; their characteristics are listed in Table 1. Prior to CIRT, 11 grade 1 patients, 6 grade 2 patients, 8 grade 3 patients, and 4 grade 4 patients were identified according to the Modified Medical Research Council Dyspnea Scale [24]. Under the CTCAE v.3.0 criteria, there were 11 grade 1 patients, 14 grade 2 patients, 4 grade 3 patients, and no grade 4 or 5 patients. Four patients were using home oxygen therapy, 4 had a history of AE prior to treatment, and 4 had a prior autoimmune disease.

\section{Treatment characteristics}

An evolving series of protocols were employed during the treatment period, the details of which are shown in Table 2. Treatment timeframes ranged between 1 day (46-50 Gy [RBE], single fraction; 13 patients), 4 days (52.8-60 Gy[RBE], 4 fractions; 14 patients), and 4 weeks (72 Gy[RBE], 16 fractions; 2 patients). The PTV ranged from $34.6 \mathrm{~mL}$ to $1319.8 \mathrm{~mL}$ (median: $145.1 \mathrm{~mL}$ ). 
Table 1 Pretreatment patient characteristics

No. of patients
Follow-up period median (range)
Gender (male/female)
Age median (range)
Performance Status (ECOG) 0/1/2/3
Smoking history (pack years) median (range)
Severity of ILD (grade 1/2/3/4)
Dyspnea evaluation before CIRT
No. of patients using home oxygen therapy
mMRC scale (grade 0/1/2/3/4)
equivalent RP grade CTCAE ver 3.0 (grade 1
/2/3/4/5)
\%FVC median (range)
\%DLco median (range)
Tumor size
T1a ( $\leq 2 \mathrm{~cm}$ )
T1b ( $2-3 \mathrm{~cm}$ )
T2a ( $3-5 \mathrm{~cm}$ )
T2b ( $\geq 5 \mathrm{~cm}$ )
Tumor location
Upper lobe (including 2 tumors in middle lobe)
Lower lobe

Histology

$\begin{array}{ll}\text { Adenocarcinoma } & 8 \\ \text { Squamous cell carcinoma } & 10 \\ \text { Unclassified non-small cell carcinoma } & 2 \\ \text { Clinically diagnosed } & 9\end{array}$

Laboratory Data

serum KL-6 (U/I) median (range)

serum SP-D (ng/l) median (range)

The time of ILD diagnosis

\begin{tabular}{ll} 
at the same time as lung cancer diagnosis & 14 \\
unknown & 1 \\
before diagnosis of lung cancer & 14 \\
$\begin{array}{l}\text { Period from ILD to cancer diagnosis (median } \\
\text { years) (range) }\end{array}$ & $4(2-13)$ \\
$\begin{array}{l}\text { Episode of acute exacerbation before treatment } \\
\text { (yes/no) }\end{array}$ & $4 / 25$ \\
$\begin{array}{l}\text { Auto-immune disease (yes/no) } \\
\text { a }\end{array}$ & $4 / 25$ \\
\hline
\end{tabular}

*data are available only for 26 patients

Abbreviations: ECOG Eastern Cooperative Oncology Group, ILD interstitial lung disease, CIRT carbon ion radiotherapy, $m M R C$ scale the modified Medical Research Council scale, FVC forced vital capacity, DLco diffusion capacity of the lung, KL-6 Krebs von den Lungen-6, SP-D surfactant protein D

29
$26.8(2.7-86.5)$
$28 / 1$
$73(62-90)$
$15 / 13 / 2 / 0$
$42(0-210)$
$9 / 10 / 6 / 4$

4

$0 / 11 / 6 / 8 / 4$

$11 / 14 / 4 / 0 / 0$

$82.3(37.9-117.6)$

$52.1(15.5-235.5)$

$34(17-63)$

2

8

14

5

3000

15

14

$2 / 23 / 4$

\#11 (left hilar lymph node)

2

9

$1167(456-2410)$

$157(31-502)$
Table 2 Treatment characteristics and dose volume analysis

\begin{tabular}{ll}
\hline Protocol & No. of pts. \\
\hline 46.0Gy (RBE) /1fr/1 day & 1 \\
48.0Gy (RBE)/1fr/1 day & 4 \\
50.0Gy (RBE)/1fr/1 day & 8 \\
52.8Gy (RBE)/4fr/4 days & 7 \\
60.0Gy (RBE)/4fr/4 days & 7 \\
72.0Gy (RBE)/16fr/4wks & 2 \\
PTV volume (ml), median (range) & $85.3(34.6-1319.8)$ \\
Dosimetric factors of the lung (\%), median (range) & \\
V5 lung & $10.5(3.0-34.8)$ \\
V10 lung & $8.6(2.6-32.8)$ \\
V15 lung & $7.1(2.2-27.1)$ \\
V20 lung & $6.2(2.0-18.0)$ \\
V25 lung & $4.6(1.8-16.8)$ \\
V30lung & $4.0(1.5-15.9)$ \\
Mean Lung Dose & $3.5(1.0-12.2)$ \\
\hline
\end{tabular}

Abbreviations: RBE relative biological effectiveness, $P T V$ planning target volume

\section{Pre- and post-treatment radiation pneumonitis and radiation-induced $\mathrm{AE}$}

We detected RP of grades 1, 2, and 3 in 9, 12, and 8 patients, respectively (Table. 3). Five patients experienced RP grade progression. Four patients experienced a single RP grade increase, from 1 to 2 or 2 to 3 , following treatment; 1 patient had a 2-degree increase, from 1 to 3 . Two patients experienced radiation-induced AE.

\section{Clinical outcomes}

The median follow-up period was 26.8 months (range, 2.7-86.5 months) for all patients. The overall survival rates at 3 and 5 years was 46.3 and $30.4 \%$ (57.2 and $42.4 \%$ for stage I disease), respectively (Fig. 2). Eighteen of 29 patients (62.1\%) showed a survival of longer than 2 years.

Table 3 RP Grade and incidence of acute exacerbation RP grade (CTCAE ver3.0)

\begin{tabular}{ll}
1 & $9(31 \%)$ \\
2 & $12(41 \%)$ \\
3 & $8(28 \%)$ \\
RP grade progression after treatment & \\
$0 / 1 / 2$ & $24 / 4 / 1$ \\
Acute exacerbation & $2(7 \%)^{a}$ \\
RP symptom onset time & \\
no symptom & $15(52 \%)$ \\
0-3 month & $9(31 \%)$ \\
$3-6$ month & $5(17 \%)$ \\
\hline
\end{tabular}

These patients are also included in RP grade 3 data Abbreviations: CTCAE Common Terminology Criteria for Adverse Events, RP radiation pneumonitis 


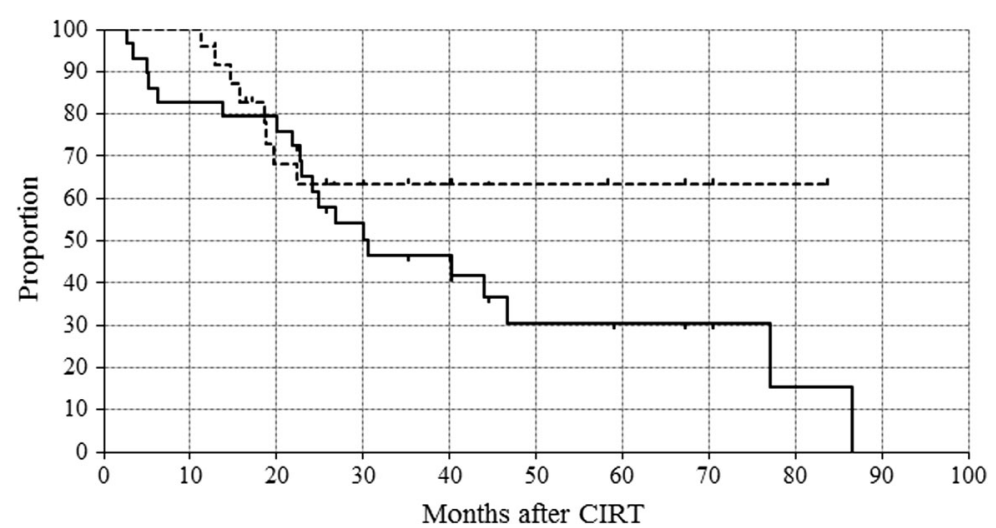

Fig. 2 Overall survival (OS) and local control rates (LCR) after carbon-ion radiotherapy. The solid line is the OS, and the dotted line is the LCR in 29 patients. The 3 - and 5 year LCRs were both $63.3 \%$ and the 3 - and 5 year OS were $46.3 \%$ and $30.4 \%$ respectively

Eighteen patients had died at the time of this writing, 10 of LC progression, 2 of other cancers (1 pancreastic, and 1 myelodysplastic syndrome), and 6 of noncancerous causes ( 3 of bacterial pneumonia, 2 of hypoxia due to progression of ILD, and 1 of gastrointestinal hemorrhage). The deaths from hypoxia resulting from ILD occurred more than 40 months after undergoing CIRT in both patients. We observed eight local recurrences; the median time between CIRT and diagnosis of local recurrence was 17.2 months. The local control rates at 3 and 5 years were both $63.3 \%$ (72.2\% for stage I disease only).

\section{Acute exacerbation}

Two patients were diagnosed with AE of their ILD postCIRT. One was a 76-year-old man with stage IA LC, and the other an 80-year-old man with stage IB LC. They were diagnosed with AE by CT scanning following hospital admission due to worsening dyspnea, which revealed ground glass opacities outside the irradiated field. Both were treated with oxygen and steroid pulse therapy. The $\mathrm{AE}$ subsided in both patients, although the man with stage IA disease died of gastrointestinal hemorrhage 1 month following admission. The patient with stage IB disease was discharged, but died of bacterial pneumonitis 5 months after radiotherapy.

\section{Radiation pneumonitis grade progression and related factors}

Table 4 shows the relationships between the RP grade progression and pretreatment clinical factors as well as dosimetric factors in the 29 patients. The lung doses of V5 and V10 were significantly associated with the occurrence of RP grade progression ( $p=0.026$ and 0.033 ). Pre-treatment serum SP-D was also found to correlate with grade progression, and was significantly lower in the grade-progressing patient population $(p=0.036)$.

\section{Discussion}

In this study, we aimed to evaluate the CIRT toxicity profile for LC patients with preexisting ILD-LC, for whom conventional radiation therapy such as SBRT is contraindicated. We evaluated 29 patients who were ineligible for surgery due to a previous diagnosis of ILD by examining the degree of RP in their lungs following treatment, as well as the degree of symptomatic change, and any AEs due to treatment.

SBRT is the typical first-line radiological intervention for early stage non-resectable LC. However, in the setting of ILD, the high risk of RP and AE limits radiotherapy to a small proportion of patients. In Yamashita et al.'s evaluation of SBRT in 117 ILD-LC patients, 9 cases of Grades 4-5 RP were noted and investigated; pretreatment blood KL-6 and SP-D levels, as well as the presence of an interstitial pneumonitis shadow, were found to be predictive of RP [25].

More recently, Ueki et al. evaluated 191 patients, 12 with mild and eight with severe cases of ILD. They found that the presence of ILD and the volume of the irradiated lung were associated with the development of grade 2 or higher RP. Furthermore, survival in the ILD group was poor compared to that in the base group [26]. Yamaguchi et al. further evaluated a group of 109 patients treated with SBRT at their institution. 16 were found to have subclinical ILD on CT and treated among these patients. Of 13 patients with post-treatment grades $2-5 \mathrm{RP}$, three were in the subclinical ILD group. Although subclinical ILD did not correlate with grades 2-5 RP in their study, the rate of extensive RP was notably high in these patients. Moreover, they reported that 2 patients with subclinical ILD developed Grade 4-5 RP [14].

The majority of the 29 patients in this trial presented with respiratory symptoms and clear interstitial opacities prior to treatment. Typically, this population of patients is offered best supportive care with no additional treatment. To our knowledge no other radiotherapy study targeted to this specific population has been performed. 
Table 4 Clinical and dosimetric factors associated with symptoms after CIRT

\begin{tabular}{|c|c|c|c|}
\hline & no grade change $(n=24)$ & grade progression $(n=5)$ & $p$-value \\
\hline \multicolumn{4}{|l|}{ Clinical factors } \\
\hline Age (yr), median (range) & $72.0(62-90)$ & $76.0(71-86)$ & 0.224 \\
\hline Performance Status (0/1/2) & $13 / 9 / 2$ & $1 / 4 / 0$ & 0.330 \\
\hline Severity of ILD (JRS) (1/2/3/4) & $9 / 7 / 4 / 4$ & $0 / 3 / 2 / 0$ & 0.434 \\
\hline mMRC scale $(1 / 2 / 3 / 4 / 5)$ & 0/9/6/5/4 & $0 / 2 / 0 / 3 / 0$ & 0.952 \\
\hline Tumor location (U/L) & $11 / 13$ & $3 / 2$ & 1.000 \\
\hline Tumor size (mm), median (range) & $34.0(17-58)$ & $31.0(22-63)$ & 0.544 \\
\hline serum KL-6, median (range) & $880.5(325-2410)$ & $544.0(466-1199)$ & 0.260 \\
\hline serum SP-D, median (range) & $152.0(60-502)$ & $105.0(31-140)$ & 0.036 \\
\hline \multicolumn{4}{|l|}{ Respiratory function, median (range) } \\
\hline$\% \mathrm{VC}$ & $90.7(43.5-130.4)$ & $86.7(64.9-107.3)$ & 0745 \\
\hline$\% \mathrm{FVC}$ & $81.8(37.9-117.6)$ & $82.7(52.8-98.7)$ & 0.820 \\
\hline FEV1.0 (L) & $2.0(1.13-2.92)$ & $2.0(1.47-2.96)$ & 1.000 \\
\hline FEV1.0/NC (\%) & $80.7(54.0-91.2)$ & $78.6(72.7-99.7)$ & 0.696 \\
\hline$\%$ DLco & $50.7(15.5-92.2)$ & $70.7(39-235.5)$ & 0.474 \\
\hline Not evaluated & 3 & 0 & \\
\hline \multicolumn{4}{|l|}{ Dose-volume metrics, median (range) } \\
\hline PTV (mL) & $82.9(34.6-345.9)$ & $88.1(52.3-1319.8)$ & 0.507 \\
\hline Total dose (Gy[RBE]), & $52.8(46-72)$ & $50.0(50-72)$ & 0.5745 \\
\hline \multicolumn{4}{|c|}{ Dosimetric factors of the lung (\%), median (range) } \\
\hline V5 & $8.7(3.0-20.6)$ & $14.2(10.9-34.8)$ & 0.026 \\
\hline V10 & $7.6(2.6-18.3)$ & $11.9(8.4-32.8)$ & 0.033 \\
\hline V15 & $5.6(2.2-13.6)$ & $8.0(7.1-27.1)$ & 0.074 \\
\hline V20 & $5.0(3.0-20.6)$ & $7.3(6.2-18.0)$ & 0.069 \\
\hline V25 & $4.2(1.8-11.0)$ & $5.4(3.3-16.8)$ & 0.237 \\
\hline V30 & $3.7(1.5-9.0)$ & $4.9(2.8-15.9)$ & 0.286 \\
\hline V35 & $3.4(1.4-8.5)$ & $4.4(2.5-5.0)$ & 0.260 \\
\hline V40 & $3.1(1.3-8.0)$ & $4.0(2.2-14.0)$ & 0.260 \\
\hline Mean Lung Dose (Gy[RBE]) & $2.8(1.0-6.4)$ & $3.8(3.0-12.2)$ & 0.075 \\
\hline
\end{tabular}

The CTCAE were used to grade post-treatment pneumonitis. However, 14 of the 29 patients in our study (48\%) presented with pretreatment grade 2-equivalent symptoms, while 4 (14\%) presented with Grade 3 equivalent symptoms. As such, we postulate that the direct grading of symptoms following radiotherapy accuracy reflect posttreatment change. We evaluated the respiratory symptom grade progression over the course of treatment, and observed progression in 5 cases. Furthermore, ILD-related AE was noted in 2 patients; both grade 3 events. Despite the extremely high risk associated with these patients, the relative lack of serious adverse events (higher than grade 3 ) was noteworthy.

Although predicting the exact prognosis of ILD is difficult owing to the varying classifications involved, ILD-LC generally has a poorer prognosis in comparison to uncomplicated LC $[4,27]$. In examining IPF specifically, Vancheri et al. and
Ley et al. noted median survival times of between 2 and 3 years for IPF-LC patients [28, 29]. Prognosis times for ILD-LC patietns remain difficult to predict, especially for those diagnosed with ILD prior to developing LC and who therefore received prior treatment. In this study, approximately half of the patients (14) were discovered to have ILD at the time of their cancer diagnosis, while the remainder received an average of 4 years of treatment prior to their LC diagnosis.

Compared to surgical patients, the average age was high in our cohort, while forced vital capacity and diffusing capacity for carbon monoxide were low. Furthermore, the patients had clear interstitial changes on imaging that disqualified them from consideration for SBRT. Compared to patients who underwent SBRT, the rate of AEs in our patients was low, while comparable overall survival rates were noted. These data are shown in the Table $5[5-9,14$, 
Table 5 Representative reported results of treatment for NSCLC with ILD

\begin{tabular}{|c|c|c|c|c|c|c|c|c|c|c|c|c|c|c|}
\hline Author & year & treatment & disease & $\begin{array}{l}\text { No of } \\
\text { all pts }\end{array}$ & $\begin{array}{l}\text { combined } \\
\text { disease }\end{array}$ & $\begin{array}{l}\text { No of pts. } \\
\text { with ILD (\%) }\end{array}$ & Age & $\begin{array}{l}\text { median follow-up } \\
\text { period (month) }\end{array}$ & $\begin{array}{l}\mathrm{AE} \\
(\%)\end{array}$ & $\begin{array}{l}\text { Mortality } \\
\text { after AE (\%) }\end{array}$ & $\begin{array}{l}3 \mathrm{yr} . \\
\text { OS (\%) }\end{array}$ & $\begin{array}{l}5 \mathrm{yr} . \\
\text { OS (\%) }\end{array}$ & $\begin{array}{l}\text { \%FVC (or } \\
\% V C \text { ) }\end{array}$ & $\%$ DLcC \\
\hline Chiyo & 2003 & surgery & NSCLC & 931 & ILD & $36(3.9)$ & 65.5 & 43.8 & $9(25)$ & $1(11.1)$ & 41.5 & 35.6 & 84.9 & - \\
\hline Mizuno & 2012 & surgery & NSCLC & 1444 & IPF & $62(4.3)$ & 71.6 & - & $\begin{array}{l}7 \\
(13.5)\end{array}$ & $6(85.7)$ & - & - & \%VC 95.3 & 51.1 \\
\hline Voltolini & 2013 & surgery & NSCLC & 775 & ILD & $37(4.8)$ & 69.3 & 26 & $\begin{array}{l}5 \\
(13.5)\end{array}$ & $3(60)$ & - & 52 & 91.4 & 59.7 \\
\hline \multirow[t]{2}{*}{ Sato } & 2014 & surgery & NSCLC & 41,742 & ILD & $1763(4.2)$ & 71 & - & $\begin{array}{l}164 \\
(9.3)\end{array}$ & $72(43.9)$ & - & - & $\begin{array}{l}\% \mathrm{VC}<80 \% \\
263\end{array}$ & - \\
\hline & 2015 & & & & & & & & & & - & 40 & $\begin{array}{l}\% \mathrm{VC}>80 \% \\
1478\end{array}$ & - \\
\hline \multirow[t]{2}{*}{ Omori } & 2015 & surgery & NSCLC & 678 & IPF & 46 & 70.9 & 34.3 & $\begin{array}{l}4 \\
(8.7)\end{array}$ & $3(75)$ & 50.9 & 22.1 & 89.8 & 70.6 \\
\hline & & & & & Non-IPF & 57 & 70.9 & & $\begin{array}{l}1 \\
(1.8)\end{array}$ & 0 & 71.5 & 53.2 & 98.5 & 71.7 \\
\hline Yamaguchi & 2013 & SBRT & $\begin{array}{l}\text { NSCLC, } \\
\text { MLT }\end{array}$ & 109 & $\begin{array}{l}\text { subclinical } \\
\text { ILD }\end{array}$ & $16(14.7)$ & 78 & 17.1 & $\begin{array}{l}2^{\mathrm{a}} \\
(12.5)\end{array}$ & $1^{\mathrm{b}}(50)$ & 48 & - & - & - \\
\hline \multirow[t]{2}{*}{ present } & 2015 & CIRT & NSCLC & 637 & ILD & $29(4.6)$ & 73 & 22.8 & $\begin{array}{l}2 \\
(6.9)\end{array}$ & 0 & 46.3 & 30.4 & 82.3 & 52.1 \\
\hline & & & $\begin{array}{l}\text { stage I } \\
\text { NSCLC }\end{array}$ & - & ILD & 22 & 73 & 22.6 & $\begin{array}{l}2 \\
(9.1 \%)\end{array}$ & 0 & 57.2 & 42.1 & 81.8 & 55.1 \\
\hline
\end{tabular}

${ }^{\mathrm{a} G r a d e} 4-5 \mathrm{RP},{ }^{\mathrm{b}}$ Grade5 RP

Abbreviations: NSCLC non-small cell lung cancer, ILD interstitial lung disease, $A E$ acute exacerbation, IPF interstitial pulmonary fibrosis, OS overall survival rate, FVC forced vital capacity, VC vital capacity, DLco diffusion capacity of the lung, MLT metastatic lung tumor, CIRT carbon ion radiotherapy, $R P$ radiation pneumotinis

30]. On the other hand, the local control rates in our study were lower than those among SBRT patients [14]. A possible explanation is that a higher percentage of our patients had T2 tumors (65.5\%). However, the local control rates in patients without ILD in our study were comparable to those in patients who underwent SBRT ([16-19]), and patients with and without ILD underwent the same treatment protocol. Hence, further explanations remain elusive.

In previous studies of LC treated with SBRT, PTV, V5,V13, V20, V25, Vprescription and mean lung dose were among the factors that showed a correlation with the severity of RP post-treatment [31-34]. In the 5 patients in our study who experienced disease progression, V5 and V10 lung doses were significantly associated with RP grade progression. However, the lung doses in these 5 patients are as low as the doses reported in the SBRT trials $[13,14,33,34]$, as heavy-ion therapy allows for improved minimization of non-target doses. Our results suggested that small differences in lung dose may significantly affect the severity of RP in symptomatic ILD patients.

With respect to the 2 cases that exhibited AE, we evaluated respiratory function tests, blood tests, dosimetric factors, and other factors to determine whether they predicted exacerbation. However, no such associations were found.

At PET pretreatment staging of the patients' cancers, highly integrated mediastinal lymph nodes were noted in 3 cases, and an accessory nerve lymph node metastasis was noted in 1 case. Among these cases, a lymph node with a minor axis greater than $10 \mathrm{~mm}$ was found in only 1 patient. Roberts et al. previously reported that, among the 7 cases of mediastinal lymph node false positives of 100 examined on PET, 4 were likely caused by inflammation in the lung parenchyma [23]. Additionally, Konishi and colleagues examined 306 mediastinal lymph nodes in 54 patients and found 7 false positives, 2 of which were caused by interstitial pneumonia [22]. Considering these reports, the 4 cases of pretreatment lymph node metastases noted on PET/CT in our ILD-LC patients may have included false positives; hence, only the primary tumors were treated. Of the 4 potential metastasis cases, none were found to have lymph node metastasis after CIRT.

As this study was retrospective, there remains the possibility that selection bias may have impacted the results, as all patients included were ineligible for surgery and SBRT because of the clear risk posed by their ILD.

\section{Conclusions}

In this study, most of patients presented with respiratory symptoms and clear interstitial opacities prior to treatment; this population had had few available treatment options outside of supportive care. Considering these unfavorable conditions, we demonstrated the efficacy and relative safety of the use of CIRT in LC patients with ILD. Dose-volume histogram analysis suggested the importance of minimizing the low-dose region, as the lung doses V5 and V10 lung doses were significantly associated with RP grade progression. As relatively few patients were evaluated in this study, a larger trial with long-term follow-up is warranted to verify these results. 


\begin{abstract}
Abbreviations
${ }^{18}$ F-FDG: [18F]-fluorodeoxyglucose; AE: Acute exacerbation; CIRT: Carbon-ion radiotherapy; CT: Computed tomography; CTCAE: Common Terminology Criteria for Adverse Events; HIMAC: Heavy Ion Medical Accelerator in Chiba; ILD: Interstitial lung disease; IPF: Interstitial pulmonary fibrosis; KL-6: Krebs von den Lungen-6; LC: Lung cancer; LCR: Local control rate; MRI: Magnetic resonance imaging; NIRS: National Institute of Radiological Sciences; NSCLC: Non-small cell lung cancer; OS: Overall survival; PET: Positron emission tomography; PTV: Planning target volume; RBE: Relative biological effectiveness; RP: Radiation pneumonitis; SBRT: Stereotactic body radiation therapy; SP-D: Surfactant protein-D
\end{abstract}

\section{Acknowledgements}

This work was supported by the Research Project with Heavy lons at NIRS-HIMAC. We are grateful to Dr. Kazuhisa Takahashi (Department of Respiratory Medicine, Juntendo University Graduate School of Medicine) and the Working Group for Lung Cancer at the NIRS.

\section{Funding}

This work did not receive any specific funding.

\section{Availability of data and materials}

The datasets used and /or analyzed during the current study are available from the corresponding author on reasonable request.

\section{Authors' contributions}

MN and NY performed the data collection, data analysis and manuscript writing. $\mathrm{KH}$ performed in statistical analysis. DKE assisted in the statistical analysis and drafting of the manuscript. MK, WT and MA participated in the patient care and target planning. KT, YT and KT made contribution to the design of this study. TM, HT, TF, and TK were involved in the general supervision of this study. All authors read and approved the final manuscript.

\section{Ethics approval and consent to participate}

All study participants provided informed consent, and we obtained general consent to the research in written form from the participant. The treatment method and procedure were approved by the ethics committees of our institution.

\section{Consent for publication}

We obtained consent for publication in written form from the patient described in Fig. 1.

\section{Competing interests}

The authors declare that they have no competing interests.

\section{Publisher's Note}

Springer Nature remains neutral with regard to jurisdictional claims in published maps and institutional affiliations.

\section{Author details \\ ${ }^{1}$ National Institute of Radiological Sciences Hospital, National Institutes for Quantum and Radiological Sciences and Technology, 4-9-1, Anagawa, Inage-ward, Chiba 263-8555, Japan. ${ }^{2}$ Department of Radiology, the University of Tokyo Hospital, Hongo, Bunkyo-ward, Tokyo 113-8655, Japan. ${ }^{3}$ Brown University Alpert Medical School, Providence, RI 02903, USA. ${ }^{4}$ Kansai Rosai Hospital, Inabaso, Amagasaki 660-8511, Japan. ${ }^{5}$ Department of Respirology, Graduate School of Medicine, Chiba University, Inohana, Chuo-ward, Chiba 260-8670, Japan. ${ }^{6}$ Chiba Foundation for Health Promotion and Disease Prevention, Shinminato, Mihama-ward, Chiba 261-0002, Japan.}

Received: 21 March 2017 Accepted: 25 August 2017 Published online: 02 September 2017

\section{References}

1. Nagai A, Chiyotani A, Nakadate T, Konno K. Lung cancer in patients with idiopathic pulmonary fibrosis. Tohoku J Exp Med. 1992;167:231-7.

2. Matsushita H, Tanaka S, Saiki Y, Hara M, Nakata K, Tanimura S, et al. Lung cancer associated with usual interstitial pneumonia. Pathol Int. 1995;45:925-32.
3. Ozawa Y, Suda T, Naito T, Enomoto N, Hoshimoto D, Fujisawa T, et al. Cumulative incidence of and predictive factors for lung cancer in IPF. Respirology. 2009;14:723-8.

4. Tomassetti S, Gurioli C, Ryu JH, Decker PA, Ravaglia C, Tantalocco P, et al. The impact of lung cancer on survival of idiopathic pulmonary fibrosis. Chest. 2015;147:157-64.

5. Chiyo M, Sekine $Y$, Iwata $T$, Tatsumi $K$, Yasufuku $K$, lyoda $A$, et al. Impact of interstitial lung disease on surgical morbidity and mortality for lung cancer: analyses of short-term and long-term outcomes. J Thorac Cardiovasc Surg. 2003;126:1141-6.

6. Mizuno Y, Iwata H, Shirahashi K, Takamochi K, Oh S, Suzuki K, et al. The importance of intraoperative fluid balance for the prevention of postoperative acute exacerbation of idiopathic pulmonary fibrosis after pulmonary resection for primary lung cancer. Eur J Cardiothorac Surg. 2012;41:e161-5.

7. Voltolini L, Bongiolatti S, Luzzi L, Bargagli E, Fossi A, Ghiribelli C, et al. Impact of interstitial lung disease on short-term and long-term survival of patients undergoing surgery for non-small-cell lung cancer: analysis of risk factors. Eur J Cardiothorac Surg. 2013;43:e17-23.

8. Sato T, Teramukai S, Kondo H, Watanabe A, Ebina M, Kishi K, et al. Impact and predictors of acute exacerbation of interstitial lung diseases after pulmonary resection for lung cancer. J Thorac Cardiovasc Surg. 2014;147:1604-11.

9. Omori T, Tajiri M, Baba T, Ogura T, Iwasawa T, Okudela K, et al. Pulmonary resection for lung cancer in patients with idiopathic interstitial pneumonia. Ann Thorac Surg. 2015;100:954-60.

10. Timmerman R, Paulus R, Galvin J, Michalski J, Straube W, Bradley J, et al. Stereotactic body radiation therapy for inoperable early stage lung cancer JAMA. 2010;303:1070-6.

11. Xia T, Li H, Sun Q, Wang Y, Fan N, Yu Y, et al. Promising clinical outcome of stereotactic body radiation therapy for patients with inoperable stage $\mathrm{I} / \mathrm{I}$ non-small-cell lung cancer. Int J Radiat Oncol Biol Phys. 2006;66:117-25.

12. Nagata Y, Hiraoka M, Shibata T, Onishi H, Kokubo M, Karasawa K, et al. Prospective trial of stereotactic body radiation therapy for both operable and inoperable T1NOMO non-small cell lung cancer: Japan clinical oncology group study JCOG0403. Int J Radiat Oncol Biol Phys. 2015;93:989-96.

13. Nagata $Y$, Hiraoka M, Mizowaki T, Narita Y, Matsuo $Y$, Norihisa $Y$, et al. Survey of stereotactic body radiationtherapy in Japan by the Japan 3-D conformal external beam radiotherapy group. Int J Radiat Oncol Biol Phys. 2009;75:343-7.

14. Yamaguchi S, Ohguri T, Ide S, Aoki T, Imada H, Yahara K, et al. Stereotactic body radiotherapy for lung tumors in patients with subclinical interstitial lung disease: the potential risk of extensive radiation pneumonitis. Lung Cancer. 2013;82:260-5.

15. Kanai T, Furusawa Y, Fukutsu K, Itsukaichi H, Eguchi-Kasai K, Ohara H. Irradiation of mixed beam and design of spread out Bragg peak for heavyion radiotherapy. Radiat Res. 1997;147:78-85.

16. Miyamoto T, Baba M, Sugane T, Nakajima M, Yashiro T, Kagei K, et al. Carbon ion radiotherapy for stage I non-small cell lung cancer using a regimen of four fractions during 1 week. J Thorac Oncol. 2007;2:916-26.

17. Sugane T, Baba M, Imai R, Nakajima M, Yamamoto N, Miyamoto T, et al Carbon ion radiotherapy for elderly patients 80 years and older with stage I non-small cell lung cancer. Lung Cancer. 2009:64:45-50.

18. Takahashi W, Nakajima M, Yamamoto N, Yamashita H, Nakagawa K, Miyamoto T, et al. A prospective nonrandomized phase I/II study of carbon ion radiotherapy in a favorable subset of locally advanced non-small cell lung cancer (NSCLC). Cancer. 2015:121:1321-7.

19. Yamamoto N, Miyamoto T, Nakajima M, et al. A dose escalation clinical trial of single-fraction carbon-ion radiotherapy for peripheral stage I non-smallcell lung cancer. J Thorac Oncol. 2017;12:673-80.

20. Japanese Respiratory Society's Committee Formulating Diagnosis and Treatment Guideline for Diffuse Lung Diseases. Revised 2nd edition guidelines for diagnosis and treatment of idiopathic interstitial pneumonias. Tokyo: Nankodo; 2011.

21. Minohara S, Kanai T, Endo M, Noda K, Kanazawa M. Respiratory gated irradiation system for heavy-ion radiotherapy. Int J Radiat Oncol Biol Phys. 2000;47:1097-103

22. Konishi J, Yamazaki K, Tsukamoto E, Tamaki N, Onodera Y, Otake T, et al. Mediastinal lymph node staging by FDG-PET in patients with non-small cell lung cancer: analysis of false-positive FDG-PET findings. Respiration. 2003:70:500-6.

23. Roberts PF, Follette DM, von Haag D, Park JA, Valk PE, Pounds TR, et al. Factors associated with false-positive staging of lung cancer by positron emission tomography. Ann Thorac Surg. 2000;70:1154-9. discussion 1159-60 
24. National Collaborating Centre for Chronic Conditions. Chronic obstructive pulmonary disease : national clinical guideline on management of chronic obstructive pulmonary disease in adults in primary and secondary care. Thorax. 2004;59(suppl 1):1-232.

25. Yamashita H, Kobayashi-Shibata S, Terahara A, Okuma K, Haga A, Wakui R, et al. Prescreening based on the presence of CT-scan abnormalities and biomarkers (KL-6 and SP-D) may reduce severe radiation pneumonitis after stereotactic radiotherapy. Radiat Oncol. 2010;5:32.

26. Ueki N, Matsuo Y, Togashi Y, Kubo T, Shibuya K, lizuka Y, et al. Impact of pretreatment interstitial lung disease on radiation pneumonitis and survival after stereotactic body radiation therapy for lung cancer. J Thorac Oncol. 2015;10:116-25.

27. Bouros D, Hatzakis K, Labrakis H. Zeibecoglou Ket al. Association of malignancy with diseases causing interstitial pulmonary changes. Chest. 2002;121:1278-89.

28. Vancheri C, Failla M, Crimi N, Raghu G. Idiopathic pulmonary fibrosis: a disease with similarities and links to cancer biology. Eur Respir J. 2010;35:496-504.

29. Ley B, Collard HR, King TE Jr. Clinical course and prediction of survival in idiopathic pulmonary fibrosis. Am J Respir Crit Care Med. 2011;183:431-40.

30. Sato T, Watanabe A, Kondo H, Kanzaki M, Okubo K, Yokoi K, et al. Long-term results and predictors of survival after surgical resection of patients with lung cancer and interstitial lung diseases. J Thorac Cordiovasc Surg. 2015;149:64-9.

31. Borst GR, Ishikawa M, Nijkamp J, Hauptmann M, Shirato H, Onimaru R, et al. Radiation pneumonitis in patients treated for malignant pulmonary lesions with hypofractionated radiation therapy. Radiother Oncol. 2009;91:307-13.

32. Barriger RB, Forquer JA, Brabham JG, Andolino DL, Shapiro RH, Henderson $M A$, et al. A dose-volume analysis of radiation pneumonitis in non-small cell lung cancer patients treated with stereotactic body radiation therapy. Int J Radiat Oncol Biol Phys. 2012;82:457-62.

33. Matsuo Y, Shibuya K, Nakamura M, Narabayashi M, Sakanaka K, Ueki N, et al. Dose-volume metrics associated with radiation pneumonitis after stereotactic body radiation therapy for lung cancer. Int J Radiat Oncol Biol Phys. 2012:83:e545-9.

34. Baker R, Han G, Sarangkasiri S, DeMarco M, Turke C, Stevens CW, et al. Clinical and dosimetric predictors of radiation pneumonitis in a large series of patients treated with stereotactic body radiation therapy to the lung. Int J Radiat Oncol Biol Phys. 2013;85:190-5.

\section{Submit your next manuscript to BioMed Central and we will help you at every step:}

- We accept pre-submission inquiries

- Our selector tool helps you to find the most relevant journal

- We provide round the clock customer support

- Convenient online submission

- Thorough peer review

- Inclusion in PubMed and all major indexing services

- Maximum visibility for your research

Submit your manuscript at www.biomedcentral.com/submit 